\title{
Sensitivity Based Voltage Support Strategy to Enhance Dynamic Stability of Islanded Microgrid
}

\author{
Nadia Afrin ${ }^{1}$, Student Member, IEEE, Monirul Islam², Student Member, IEEE, Junwei Lu' ${ }^{1}$, Senior Member, IEEE, \\ and Fuwen Yang ${ }^{1, \dagger}$, Senior Member, IEEE
}

\begin{abstract}
This paper presents a new voltage support (VS) strategy for photovoltaic (PV) systems based on voltage sensitivity analysis to enhance the dynamic voltage stability (DVS) of islanded microgrids (MGs). Every network contains different characteristics. In particular, MGs have unique features compared to utility grids such as high resistance to reactance ratio $(R / X)$. Consequently, injection of both active and reactive current is likely to improve the DVS of the islanded MG. Therefore, instead of engaging the entire capacity to reactive current injection which is performed in conventional voltage support strategy, both the active and reactive current is injected in the proposed strategy. On contrary, without considering the fact that each network contains distinctive characteristics, other strategies with both active and reactive current injection provide fixed active and reactive current which are uniform for all networks. To convey the network distinctive features on VS strategy, voltage sensitivity subject to active and reactive current injection is used in the proposed strategy. As a consequence, unlike other fixed active and reactive current strategies, the proposed strategy provides unique combination of active and reactive current for every network. The concept and derivation of the proposed strategy is clearly described. Network sensitivity analysis is carried out to determine the voltage sensitivity coefficients. The effectiveness of the proposed strategy is theoretically demonstrated and also compared with other strategies. Case studies are carried out on two different islanded MGs. The results show that the proposed voltage support strategy is capable of enhancing the DVS of islanded MGs by mitigating a possible instability, and by restoring the voltage faster than other strategies.
\end{abstract}

Index Terms-Sensitivity analysis, dynamic voltage stability, photovoltaic system, $\mathrm{R} / \mathrm{X}$ ratio, voltage support strategy.

\section{INTRODUCTION}

The demand for renewable energy (RE) in the form of distributed generators (DGs) originated the notion of $\mathrm{MG}$, which can be operated in grid-connected or islanded mode [1]. To facilitate the isolated areas, such as oceanic islands and remote villages with adequate electricity supply, RE dominated islanded MGs have been attracting significant attention. Nevertheless, the unique characteristics of islanded MGs, such as smaller system size, high penetration of $\mathrm{RE}$ sources, higher $\mathrm{R} / \mathrm{X}$ ratio, limited short-circuit capacity result in particular stability issues [2], [3]. Therefore, attainment of secured operation for MGs becomes more challenging compared to conventional power systems [4], [5]. Among various stability issues, maintaining dynamic voltage stability of RE-dominated MGs following severe disturbances is a major challenge, because of the poor short-circuit current contribution of inverter-based RE sources [2], [6].

Moreover, MGs contain a significant amount of induction motor (IM) in the form of both residential and commercial loads [2], [7]. The transition from normal operating mode to stalling mode of IMs, when a fault occurs, causes extra power demand which is around three to five times of rated power [8].

\footnotetext{
${ }^{1}$ N. Afrin, F. Yang, and J. Lu are with the Griffith School of Engineering, Griffith University, QLD 4222, Australia.

${ }^{2}$ M. Islam, Power System Engineer, APD Engineering, Brisbane, Australia.

†Corresponding author's Email: fuwen.yang@griffith.edu.au.
}

Additionally, the short-circuit current contribution of inverterbased generators is less compared to synchronous generators [2], [9]. As a consequence, recovering the voltage after the occurrence of fault at inverter dominated MGs is more challenging than for conventional grid [10]. Accordingly, the DVS issue of inverter dominated islanded MGs gained much attention.

The conventional methods of improving DVS are (a) the installation of static volt ampere reactive compensators (SVCs), and (b) shedding loads in the most severe cases [11]. Note that the implementation of SVCs is costly. On the other hand, load shedding is not a favorable approach from the perspective of the customer. Since the penetration level of PV systems is increasing and, most importantly, they are being placed in a distributed pattern. An effective way to improve the DVS is to provide voltage ride-through capability as well as VS from the PV system. Therefore, several grid codes for PV systems are imposed by network service providers in different countries for these ancillary services [12], [13], [14]. However, those codes are assigned for the conventional grid-connected PVs. To the best of the authors' knowledge, no specification for islanded MG condition exists, although MG with a highly penetrated PV system is a growing sector. Theoretically, voltage tends to increase with the injection of power. In conventional power systems, this happens with reactive power injection predominantly because of negligible resistive line parameters [15]. Therefore, conventionally the VS strategy with constant peak current control has been designed to focus on the precedence of reactive current [16], [17], [18]. In the conventional strategy, the active current is considered as a subordinate component and decreased to increase the reactive current injection. Consequently, only reactive current is injected for a range of voltage dips, which results in zero active current.

Furthermore, injection of both active and reactive current is an effective strategy to enhance the DVS of the network, especially for medium and low voltage networks. Several researches have been conducted with the objective of injecting both active and reactive current as voltage support to improve the DVS [6], [19], [20]. In [20], more reactive current has been injected by reducing the active current as the total current is constant, which is similar to the conventional strategy. Thereafter, after a certain voltage drop, the current capacity is equally divided between active and reactive current components. In [6], initially, active current is maintained to the pre-fault value and the reactive current is increased until the total current reached the maximum capacity of the inverter. After that, to limit the total current within the maximum value, active current is decreased. Finally, the maximum capacity is occupied by active and reactive current components uniformly during severe voltage drop. In [19], the active current component is designed to be increased without violating maximum capacity during the initial stage of voltage dip, and the reactive current is injected as per the conventional strategy. Later, reactive current is limited to the rated value while 
the margin of the inverter is used for active current injection.

MG networks convey distinct and inherent characteristics, such as distributed patterns of generators and considerable resistive line parameter [2], [21]. Hence, only reactive current injection such as strategies proposed in [16], [17], [18], are not the most effective approach to alleviate probable dynamic voltage instability. Furthermore, the existing strategies proposed in [6], [19], [20] have provided fixed and predetermined active and reactive current, which are same for all networks, although each network conveys unique characteristics and conditions. It needs to be mentioned that DVS can be affected by those features of the networks. Therefore, injection of same active and reactive current during the faults for every MG might not be most effective way to improve the DVS. Consequently, it is necessary to consider the characteristics of the MG to propose a new VS strategy. However, as discussed previously, the existing VS strategies have not considered the network characteristics. Furthermore, inverters have strict current limitations. Hence, the proportion of active and reactive current injection should be used effectively with the aim of maximized DVS improvement. Considering all the aforementioned drawbacks, a new VS strategy is proposed in this paper which has the following features: (1) comprehension of network characteristics (2) adaptivity with the change of the network and (3) capability to provide the improved DVS of islanded MG.

To overcome the drawbacks in the existing works, the influence of the network characteristics on the DVS of the network is attained through sensitivity analysis of the voltage in the proposed strategy. Voltage sensitivity analysis conveys the correlation of the voltage to active and reactive current injection. In this approach, the influence of network characteristics on the voltage can be quantified and conceptualized. Finally, the proposed voltage support strategy is designed based on the sensitivity analysis. The contribution of this paper can be summarized as: (a) determination of voltage sensitivity with respect to active and reactive current injection, (b) network dependent allocation of active and reactive current component is conceptualized through voltage sensitivity analysis, and (c) the current capacity of the inverter is effectively used to enhance the DVS of islanded MGs. As a consequence of voltage sensitivity integration, the proposed strategy is proficient in distinguishing between different networks and can inject an appropriate amount of active and reactive current to enhance the DVS.

The paper is arranged as follows: Section II contains PV system modelling. Section III outlines the proposed VS strategy. To validate the proposed strategy, case studies are conducted in two islanded MGs and the results and analyses are presented in Section IV. Finally, section V narrates the conclusions and contributions of the paper.

\section{PV System MODELling}

Fig. 1a illustrates the three-phase PV inverter connected to MG through a LC filter. In Fig. 1a, $V_{P V}, C_{d c}, L_{f}, C_{f}, V_{g}$, and $I_{g}$ are the voltage across the PV panel and dc link capacitor, filter inductance, filter capacitance, terminal voltage, and injected current from the inverter, respectively. The control structure of the inverter is demonstrated in Fig. 1b. The measured phase voltages $\left(V_{g a b c}\right)$ and current $\left(I_{g a b c}\right)$ are converted to the dq components and injected to the current controller. The reference current component is determined depending on the system condition. At normal

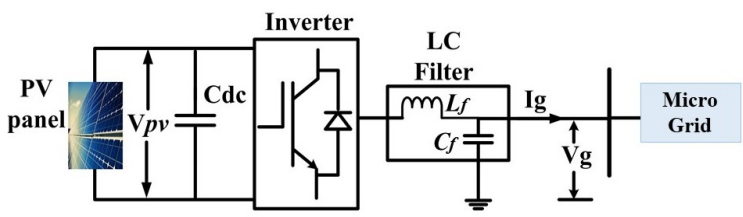

(a)

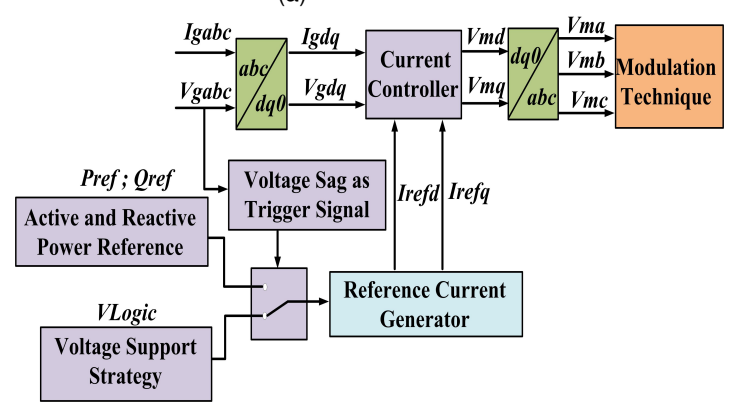

(b)

Fig. 1. (a) Schematic diagram, and (b) control structure of a three-phase PV inverter connected to the islanded MG.

operating condition, the reference active $\left(I_{\text {refd }}\right)$ and reactive $\left(I_{r e f q}\right)$ current components are determined by (1), where $V_{g d}$ and $V_{g q}$ are measured voltage elements, and $P_{r e f}$ and $Q_{r e f}$ are reference power elements which are set by the grid operator. Under faulty conditions, $I_{r e f d}$ and $I_{r e f q}$ are evaluated according to the voltage support strategy.

$$
\left[\begin{array}{c}
I_{r e f d} \\
I_{r e f q}
\end{array}\right]=\frac{2}{3\left(V_{g d}^{2}+V_{g q}^{2}\right)}\left[\begin{array}{cc}
V_{g d} & V_{g q} \\
V_{g q} & -V_{g d}
\end{array}\right]\left[\begin{array}{c}
P_{r e f} \\
Q_{r e f}
\end{array}\right]
$$

\section{Proposed Sensitivity Based Voltage Support STRATEGY}

\section{A. Concept of the proposed strategy}

As mentioned in the literature section, previously presented voltage support strategies have not considered the voltage sensitivity with respect to active $\left(I_{d}\right)$ and reactive $\left(I_{q}\right)$ current injection. As a consequence, the allocation of $I_{d}$ and $I_{q}$ current component is same for all networks. Conversely, every network has individual characteristics, for example MGs contain distributed patterns of generators as well as high $\mathrm{R} / \mathrm{X}$ line ratio, which can affect the voltage stability. Therefore, injection of same active and reactive current during the faults for every network might not be most effective to improve the DVS. Considering this, a sensitivity based VS strategy is developed. To determine the maximized voltage increment at the terminal point of PV inverter, an objective function has been defined in [20],[22] as (2) where the change in the terminal voltage with respect to $I_{d}$ and $I_{q}$ has been approximately expressed.

$$
\begin{array}{r}
\text { Maximise } f=\Delta V \cong \sigma_{V I_{d}} I_{d}+\sigma_{V I_{q}} I_{q} \\
\text { Subject to } V_{g} \leq V_{t}, I_{m}=\sqrt{I_{d}^{2}+I_{q}^{2}}
\end{array}
$$

where, $I_{m}$ is the maximum injected current, and $\sigma_{V I_{d}}$ and $\sigma_{V I_{q}}$ are the sensitivity coefficients of the terminal voltage of the inverter with respect to $I_{d}$ and $I_{q}$, respectively, which can be defined as follows:

$$
\sigma_{V I_{d}}=\frac{\partial V}{\partial I_{d}} ; \quad \sigma_{V I_{q}}=\frac{\partial V}{\partial I_{q}}
$$

The voltage sensitivity coefficients represent the variation of voltage at a bus with respect to the variation of injected active or reactive current and convey the correlation between the current components and voltage increment. The coefficients will be 
unique for each network condition. Therefore, it is important to consider these coefficients in voltage support strategies for achieving enhanced DVS. Hence, a new voltage support strategy based on voltage sensitivity coefficients is proposed as follows:

$$
\begin{array}{r}
I_{\text {ref } q}= \begin{cases}I_{m} \lambda\left(1-V_{g}\right) & \text {; if } 1-\frac{1}{\lambda \sqrt{\mu^{2}+1}} \leq V_{g} \leq V_{t} \\
\frac{\sigma_{V I_{q}}}{\sqrt{\sigma_{V I_{d}}{ }^{2}+\sigma_{V I_{q}}{ }^{2}} I_{m}} & \text {; if } V_{g}<1-\frac{1}{\lambda \sqrt{\mu^{2}+1}}\end{cases} \\
I_{\text {refd }}= \begin{cases}I_{m} \sqrt{1-\lambda^{2}\left(1-V_{g}\right)^{2}} & \text {; if } 1-\frac{1}{\lambda \sqrt{\mu^{2}+1}} \leq V_{g} \leq V_{t} \\
\frac{\sigma_{V I_{d}}}{\sqrt{\sigma_{V I_{d}}{ }^{2}+\sigma_{V I_{q}}^{2}} I_{m}} & \text {; if } V_{g}<1-\frac{1}{\lambda \sqrt{\mu^{2}+1}}\end{cases}
\end{array}
$$

Where, $\mu$ represents the ratio between $\sigma_{V I_{d}}$ and $\sigma_{V I_{q}}$.

\section{B. Framework of the proposed strategy}

Let us consider a simplified network, illustrated in Fig. 2, to determine the voltage sensitivity coefficients where PV system is connected at bus $i$. The injected active and reactive power from the PV system can be calculated as follows:

$$
\begin{aligned}
& P_{i}=V_{i} \sum_{k=1}^{n} V_{k} Y_{i k} \cos \left(\delta_{i}-\delta_{k}-\theta_{i k}\right) \\
& Q_{i}=V_{i} \sum_{k=1}^{n} V_{k} Y_{i k} \sin \left(\delta_{i}-\delta_{k}-\theta_{i k}\right)
\end{aligned}
$$

From (7) and (8), the active and reactive current elements can be determined as follows:

$$
\begin{aligned}
& I_{d_{i}}=\frac{P_{i}}{V_{i}}=\sum_{k=1}^{n} V_{k} Y_{i k} \cos \left(\delta_{i}-\delta_{k}-\theta_{i k}\right) \\
& I_{q_{i}}=\frac{Q_{i}}{V_{i}}=\sum_{k=1}^{n} V_{k} Y_{i k} \sin \left(\delta_{i}-\delta_{k}-\theta_{i k}\right)
\end{aligned}
$$

The linearization of the aforementioned equations can be done by (11) to find the sensitivity coefficients, where elements of $J$ matrix represents sensitivity factors.

$$
\left[\begin{array}{l}
\Delta I_{d} \\
\Delta I_{q}
\end{array}\right]=\left[\begin{array}{ll}
J_{I_{d} \delta} & J_{I_{d} V} \\
J_{I_{q} \delta} & J_{I_{q} V}
\end{array}\right]\left[\begin{array}{c}
\Delta \delta \\
\Delta V
\end{array}\right]
$$

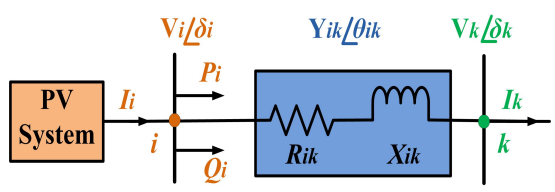

Fig. 2. Simplified model for voltage sensitivity calculation.

To find the absolute voltage sensitivity with respect to $I_{d}$ and $I_{q}$, injection of only one component is considered at a time. At first, $\sigma_{V I_{d}}$ is determined. In this situation, from (11), the expressions of active current deviation and angle are formulated as (12) and (13), respectively.

$$
\begin{gathered}
\Delta I_{d}=J_{I_{d} \delta} \Delta \delta_{d}+J_{I_{d} V} \Delta V \\
\Delta \delta_{d}=-\Delta V J_{I_{q} V} J_{I_{q} \delta}
\end{gathered}
$$

Substituting (13) into (12):

Where,

$$
\Delta I_{d}=\Delta V\left\{J_{I_{d} V}-J_{I_{d} \delta} J_{I_{q} V} J_{I_{q} \delta}{ }^{-1}\right\}=\Delta V J_{R_{d}}
$$

The elements of sensitivity index are as follows:

$$
\begin{gathered}
J_{I_{d} V}=\frac{\partial I_{d_{i}}}{\partial V_{k}}=\sum_{k=1}^{n} Y_{i k} \cos \left(\delta_{i}-\delta_{k}-\theta_{i k}\right) \\
J_{I_{d} \delta}=\frac{\partial I_{d_{i}}}{\partial \delta_{k}}=\sum_{k=1}^{n} V_{k} Y_{i k} \sin \left(\delta_{i}-\delta_{k}-\theta_{i k}\right)
\end{gathered}
$$

$$
\begin{gathered}
J_{I_{q} V}=\frac{\partial I_{q_{i}}}{\partial V_{k}}=\sum_{k=1}^{n} Y_{i k} \sin \left(\delta_{i}-\delta_{k}-\theta_{i k}\right) \\
J_{I_{q} \delta}=\frac{\partial I_{q_{i}}}{\partial \delta_{k}}=-\sum_{k=1}^{n} V_{k} Y_{i k} \cos \left(\delta_{i}-\delta_{k}-\theta_{i k}\right)
\end{gathered}
$$

Inserting the sensitivity indexes in (15) and (14) results the voltage sensitivity coefficient to active current as shown in (20).

$$
\sigma_{V I_{d}}=\frac{\partial V}{\partial I_{d}}=\left[\sum_{k=1}^{n} Y_{i k}\left\{\frac{1}{\cos \left(\delta_{i}-\delta_{k}-\theta_{i k}\right)}\right\}\right]^{-1}
$$

Similarly, the angle and reactive current deviation in the case of absolute sensitivity coefficient of reactive current can be determined by (21) and (22), respectively.

$$
\begin{gathered}
\Delta \delta_{q}=-\Delta V J_{I_{d} V} J_{I_{d} \delta}{ }^{-1} \\
\Delta I_{q}=\Delta V\left\{J_{I_{q} V}-J_{I_{q} \delta} J_{I_{d} V} J_{I_{d} \delta}{ }^{-1}\right\}=\Delta V J_{R_{q}}
\end{gathered}
$$

Where,

$$
J_{R_{q}}=\left\{J_{I_{q} V}-J_{I_{q} \delta} J_{I_{d} V} J_{I_{d} \delta}{ }^{-1}\right\}
$$

Putting the sensitivity indexes in (22) and (23) results the voltage sensitivity coefficient to reactive current as shown in (24).

$$
\sigma_{V I_{q}}=\frac{\partial V}{\partial I_{q}}=\left[\sum_{k=1}^{n} Y_{i k}\left\{\frac{1}{\sin \left(\delta_{i}-\delta_{k}-\theta_{i k}\right)}\right\}\right]^{-1}
$$

\section{Significance of coefficients in the proposed strategy}

The significance of considering the sensitivity coefficients in the VS strategy to improve the DVS is assessed in this section. For analysis, the modified IEEE 4 bus distribution system is considered as islanded MG network which is named $\mathrm{MG}_{-} 1$ and shown in Fig. 3. The sensitivity coefficients derived in section III-B are determined at bus 4 with the variation of line $\mathrm{R} / \mathrm{X}$ ratio and illustrated in Fig. 4. As can be seen in Fig. 4, $\sigma_{V I_{d}}$ tends to increase whereas $\sigma_{V I_{q}}$ follows the declination with the increment of $\mathrm{R} / \mathrm{X}$ ratio. Even for low $\mathrm{R} / \mathrm{X}$ ratio, the system contains a value for $\sigma_{V I_{d}}$ other than zero. From this observation, it can be noted that the system contains different value of $\sigma_{V I_{d}}$ and $\sigma_{V I_{q}}$ for different condition of network parameters. As a consequence, the consideration of sensitivity coefficients in the voltage support strategy introduces the ability to adapt with the network condition variation, which would ultimately provide an enhanced DVS for different networks.

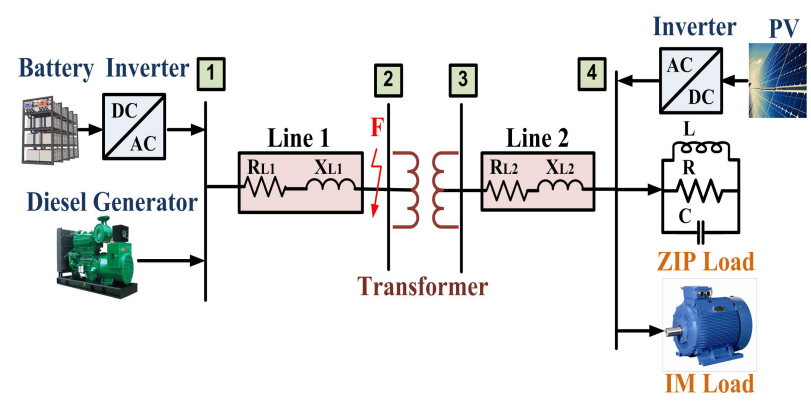

Fig. 3. Modified IEEE 4 bus distribution system as Microgrid system_1.

\section{Comparison with other strategies}

The active and reactive current contribution by the PV system during the faulty condition is identified and compared for various VS strategies in this section. Current injection by different strategies are outlined in Table I, where, $I_{r}, I_{m}, V_{g}, V_{t}, \phi$, and $\lambda$ are the rated current, maximum tolerable current capacity of the inverter, grid voltage in pu, the threshold voltage in pu, 
TABLE I

COMPARISON OF DIFFERENT STRATEGIES

\begin{tabular}{|c|c|c|c|}
\hline Strategies & Adaptability & $I_{r e f q}$ & $I_{\text {refd }}$ \\
\hline Case_I [17] & No & $\begin{array}{ll}I_{m} \lambda\left(1-V_{g}\right) & ; \text { if }(1-1 / \lambda) \leq V_{g} \leq V_{t} \\
I_{m} & \text {; if } V_{g}<(1-1 / \lambda)\end{array}$ & $\begin{array}{ll}I_{m} \sqrt{1-\lambda^{2}\left(1-V_{g}\right)^{2}} & \text {; if }(1-1 / \lambda) \leq V_{g} \leq V_{t} \\
0 & \text {; if } V_{g}<(1-1 / \lambda)\end{array}$ \\
\hline Case_II [19] & No & $\begin{cases}I_{r} \lambda\left(1-V_{g}\right) & \text {; if }(1-1 / \lambda) \leq V_{g} \leq V_{t} \\
I_{r} & \text {; if } V_{g}<(1-1 / \lambda)\end{cases}$ & $\begin{cases}I_{r} / V_{g} & \text {; if } I g \leq I_{m} \\
I_{r} \sqrt{\phi^{2}-\lambda^{2}\left(1-V_{g}\right)^{2}} & \text {; if } I_{g}>I_{m}\end{cases}$ \\
\hline Case_III [20] & No & $\begin{cases}I_{m} \lambda\left(1-V_{g}\right) & ; \text { if } \frac{\sqrt{2} \lambda-1}{\sqrt{2} \lambda} \leq V_{g} \leq V_{t} \\
I_{m} / \sqrt{2} & \text {; if } V_{g}<\frac{\sqrt{2} \lambda-1}{\sqrt{2} \lambda}\end{cases}$ & $\begin{array}{ll}I_{m} \sqrt{1-\lambda^{2}\left(1-V_{g}\right)^{2}} & \text {; if } \frac{\sqrt{2} \lambda-1}{\sqrt{2} \lambda} \leq V_{g} \leq V_{t} \\
I_{m} / \sqrt{2} & \text {; if } V_{g}<\frac{\sqrt{2} \lambda-1}{\sqrt{2} \lambda}\end{array}$ \\
\hline Case_IV [6] & No & $\begin{cases}I_{m} \lambda\left(1-V_{g}\right) & ; \text { if } \frac{\sqrt{2} \lambda-1}{\sqrt{2} \lambda} \leq V_{g} \leq V_{t} \\
I_{m} / \sqrt{2} & ; \text { if } V_{g}<\frac{\sqrt{2} \lambda-1}{\sqrt{2} \lambda}\end{cases}$ & $\begin{cases}I_{r} & ; \text { if } 1-\frac{\sqrt{\phi^{2}-1}}{\lambda \phi} \leq V_{g} \leq V_{t} \\
I_{m} \sqrt{1-\lambda^{2}\left(1-V_{g}\right)^{2}} & ; \text { if } \frac{\sqrt{2} \lambda-1}{\sqrt{2} \lambda} \leq V_{g} \leq 1-\frac{\sqrt{\phi^{2}-1}}{\lambda \phi} \\
I_{m} / \sqrt{2} & \text {; if } V_{g}<\frac{\sqrt{2} \lambda-1}{\sqrt{2} \lambda}\end{cases}$ \\
\hline $\begin{array}{c}\text { Case_V } \\
\text { (Proposed) }\end{array}$ & Yes & $\begin{cases}I_{m} \lambda\left(1-V_{g}\right) & \text {; if } 1-\frac{1}{\lambda \sqrt{\mu^{2}+1}} \leq V_{g} \leq V_{t} \\
\frac{\sigma_{V I q}}{\sqrt{\sigma_{V I_{d}}{ }^{2}+\sigma_{V I_{q}}^{2}}} I_{m} & \text {; if } V_{g}<1-\frac{1}{\lambda \sqrt{\mu^{2}+1}}\end{cases}$ & $\begin{array}{ll}I_{m} \sqrt{1-\lambda^{2}\left(1-V_{g}\right)^{2}} & \text {; if } 1-\frac{1}{\lambda \sqrt{\mu^{2}+1}} \leq V_{g} \leq V_{t} \\
\frac{\sigma_{V I_{d}}}{\sqrt{\sigma_{V I_{d}}^{2}+\sigma_{V I q}^{2}}} I_{m} & \text {; if } V_{g}<1-\frac{1}{\lambda \sqrt{\mu^{2}+1}}\end{array}$ \\
\hline
\end{tabular}

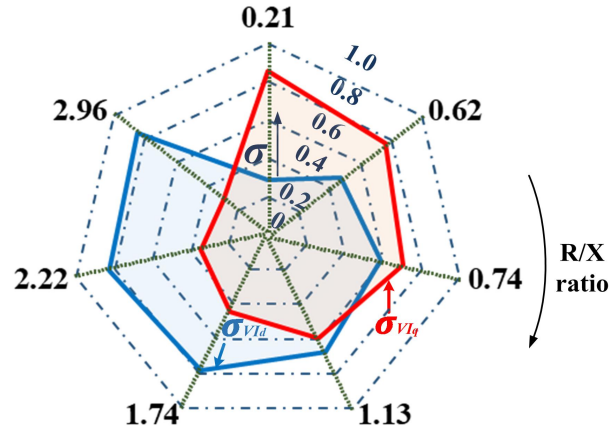

Fig. 4. Voltage sensitivity coefficients at bus 4 of MG_1 with R/X ratio variation.

maximum current margin factor of the PV inverter, and reactive current scaling factor, respectively. Note that, $\phi$ is imposed for momentary application of inverter [6], [19], [23], can be defined as $I_{m} / I_{r} \geq 1$ which is considered to be 1.25 in this paper, and $\lambda$ is set to $\geq 3$ according to the E.ON grid code [16]. For easy comprehension, strategies are termed as different cases. Table I shows that different strategies provide distinct combinations of current injection for various voltage levels. The most remarkable feature from this comparison can be notified is that only the proposed strategy has the adaptability with the network variation. The reason for this feature is that the proposed strategy allocates the active and reactive current components based on the voltage sensitivity coefficients which vary with the variation of the networks. On the contrary, other strategies provide a fixed allocation of current components for every network.

$$
\begin{gathered}
f_{\text {Case } \_}=\sigma_{V I_{q}} * I_{m} \\
f_{\text {Case_II }}=I_{r}\left(\sigma_{V I_{d}} \sqrt{\phi^{2}-1}+\sigma_{V I_{q}}\right) \\
f_{\text {Case_III\&IV }}=\frac{I_{m}}{\sqrt{2}}\left(\sigma_{V I_{d}}+\sigma_{V I_{q}}\right) \\
f_{\text {Case }-V}=I_{m} \sqrt{\sigma_{V I_{d}}^{2}+\sigma_{V I_{q}}^{2}}
\end{gathered}
$$

To demonstrate the difference between the strategies in further, the objective function for maximized voltage increment as defined
(2) is determined for the VS strategies in (25) to (28), and demonstrated with the variation of sensitivity coefficients in Fig. 5. As can be seen, the voltage increment for the proposed strategy is significantly different from those of others. Voltage is more likely to decrease when $\sigma_{V I_{d}}$ is higher compared to $\sigma_{V I_{q}}$ for Case_I which can be seen from Fig. 5a. In Case_II (Fig. 5b), the pattern is also similar to Case_I. However, the slope of the reduction is not sharp like Case_I. Since both Case_III and Case_IV inject the same amount of $I_{d}$ and $I_{q}$ after a certain voltage level, they demonstrate the same functional value, which is shown in Fig. 5c. Both Case_III and Case_IV show better performance compared to Case_I and Case_II. However, yet the functional value of Case_III and Case_IV varies with the variation of sensitivity coefficients. In contrast, unlike other strategies, the voltage incremental function for the proposed strategy is maintained with persistence, which is the highest among all strategies. This is because of the consideration of sensitivity coefficients into the allocation of current components, which is the highest among all strategies. In the following section, in addition to the time-domain simulation, DVS is also evaluated by voltage sag severity index (VSSI), presented in [24] and determined as follows:

$$
V S S I=\left\{\begin{array}{ll}
0 & \text {,if } V(t)>V_{t} \\
\sum\left(V_{t}-V(t)\right) \Delta t & \text {,if } V(t) \leq V_{t}
\end{array}+ \begin{cases}0 & \text {, if } T_{1}<10 \\
10 & \text {, otherwise }\end{cases}\right.
$$

where, $V(t), \Delta t$, and $T_{1}$ is load bus voltage (p.u.) at time $t$, step size of simulation, and the duration that the load bus voltage is below $V_{t}$ (threshold voltage), respectively.

\section{Case Studies and Result Analysis}

To validate the effectiveness of the proposed sensitivity based voltage support strategy, two islanded microgrids dominated by PV generators are developed and investigations are carried-out for various scenarios. The obtained results are compared with other strategies.

\section{A. Microgrid System_1}

Fig. 3 demonstrates the overall structure of the microgrid system_1 (MG_1) consisting of two PV system of 0.4 MVA each, 


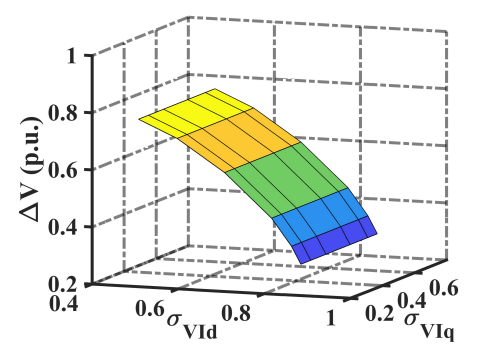

(a)

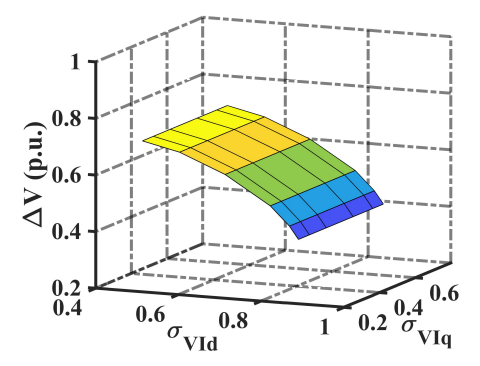

(b)

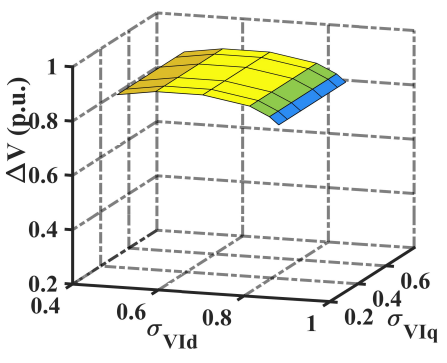

(c)

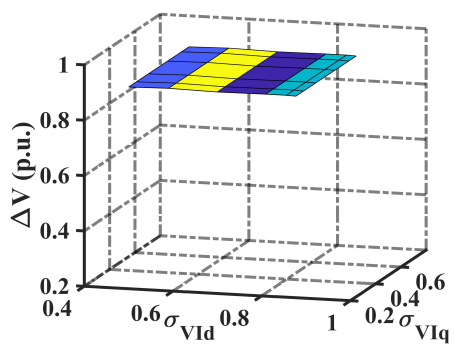

(d)

Fig. 5. Voltage increment with the variation of voltage sensitivity coefficients of MG_1 by (a) Case_I, (b) Case_II, (c) Case_III and Case_IV, and (d) Case_V.

a battery energy storage system of 1.4 MVA, and a backup diesel generator of 1.3 MVA. In MGs, the induction motor load could be as high as $50 \%$ or even more of the total load depending on the weather, seasons, location, and type of the network [7]. To obtain a practically relevant setup, 1.0 MVA of total load is considered as a combination of 50\% ZIP load (constant impedance, constant current and constant power) and 50\% IM load. Details of loadings and IM parameters are given in Table III and Table II, respectively, of Appendix. Different scenarios such as various characterized faults including assymetrical faults, $\mathrm{R} / \mathrm{X}$ ratio variation, and different power factor operation are investigated. Five strategies are compared in each scenario to examine the effectiveness of the proposed strategy in enhancing the DVS of the islanded MG.

1) Different symmetrical faults: Two three-phase to ground faults (3LG) are imposed at point $F$ as shown in Fig. 3. First fault (Fault_I) is imposed for $0.35 \mathrm{~s}$ with fault resistance $40 \Omega$. The voltage recovery time (VRT) is calculated in order to compare the effectiveness of the proposed strategy with other strategies. Fig. $6 \mathrm{a}$ to $6 \mathrm{~d}$ demonstrate the per-unit value of the dynamic voltage profile at bus 4 , total injected current from PV, and the magnitude of Id and Iq of injected current for Fault_I, respectively. It can be seen from Fig. 6a that the strategies, Case_II to Case_V, which inject both active and reactive current, are capable of recovering the voltage within the considered time frame. In contrast, Case_I injects only reactive current, and experiences voltage instability. However, it can be noted that though Case_II to Case_V have successfully recovered the voltage to stable condition, Case_V provided the fastest recovery. To be specific, the faster voltage recovery by Case_V compared to Case_II, Case_III, and Case_IV are $0.3 s, 0.1 s$, and $0.2 s$, respectively. This fastest voltage recovery by the proposed strategy occurred because of the substantial difference between the proposed and other strategies in injecting $I_{d}$ and $I_{q}$, which can be observed from Fig. $6 \mathrm{c}$ and $6 \mathrm{~d}$. As seen, Case_I injects only reactive current component during voltage sag as it is designed in this pattern. Hence, Case_I experiences highest voltage depth during fault, and finally voltage instability occurred. For Case_II to Case_V, although all these strategies provide both active and reactive current components, significant differences exist in the allocation of current components. From Fig. $6 \mathrm{c}$ and $6 \mathrm{~d}$, it can be observed that the proposed strategy provides the highest $I_{d}$ and lowest $I_{q}$ among all strategies during the fault, which are determined based on voltage sensitivity analysis. After the fault clearance, the pattern of $I_{d}$ injection is the same as other cases although magnitude is different, except in Case_IV where $I_{d}$ is maintained at the pre-fault value. Likewise, the pattern of $I_{q}$ is also the same for all with diverse magnitudes. Although the current component combination for Case_ $\mathrm{V}$ is different from

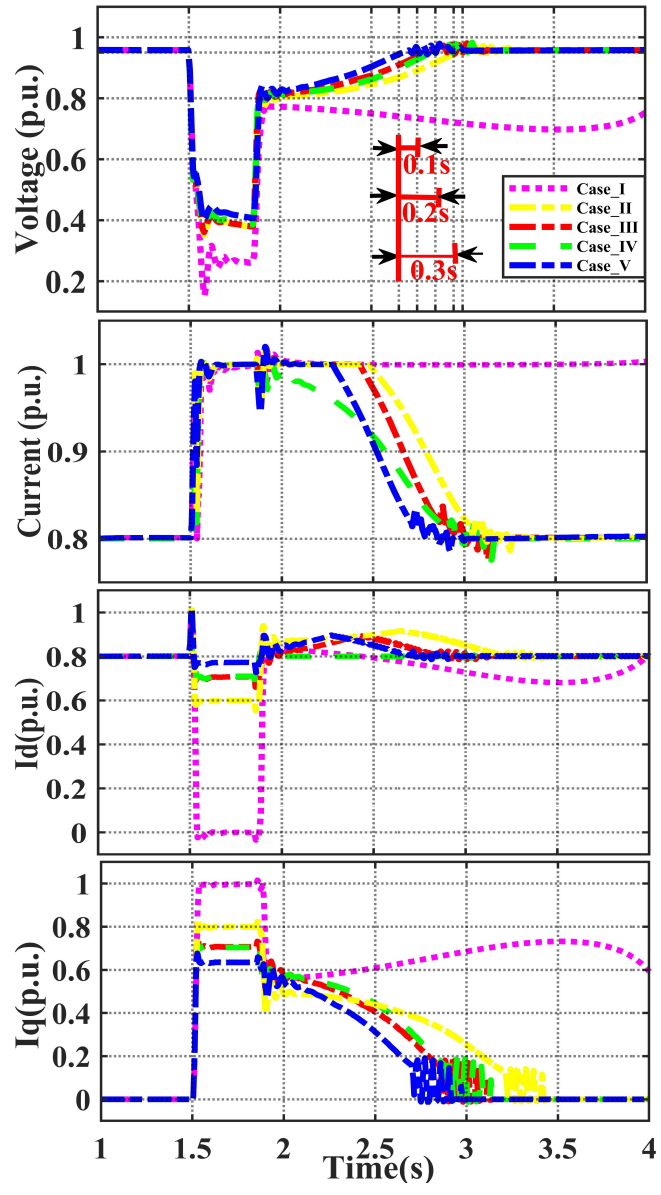

(a)

(b)

Fig. 6. (a) Dynamic voltage profile at bus 4 of $M G_{-}$1, (b) total injected current from PV, and the magnitude of (c) I_d and (d) I_q of injected current for Fault_I.

other cases, it must be noted that the total injected current never exceeds the maximum current as like other strategies which can be seen from Fig. 6b. Finally, the fastest voltage recovery result is achieved with the proposed strategy, which is Case_V, compared to others for Fault_I scenario.

In order to examine the effectiveness of the proposed strategy in the case of a shallow fault, another 3LG fault (Fault_II) with a duration of $0.5 \mathrm{~s}$ is considered. The dynamic voltage profile and the injected power for Fault_II are illustrated in Fig. 7a to 7c. The comparative portraiture of the performances for restoring the dynamic voltage profile by all cases is outlined in Fig. 7a. As seen, notable differences exist between the VRT of Case_V and other Cases. In this scenario, Case_III, Case_IV, and Case_V are capable of restoring the voltage while Case_I and Case_II have experienced voltage instability. However, the VRT of Case_V 


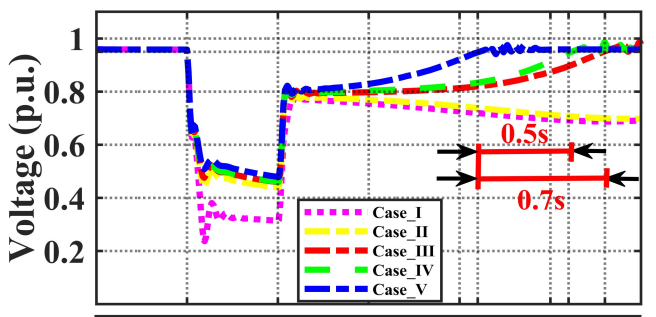

(a)

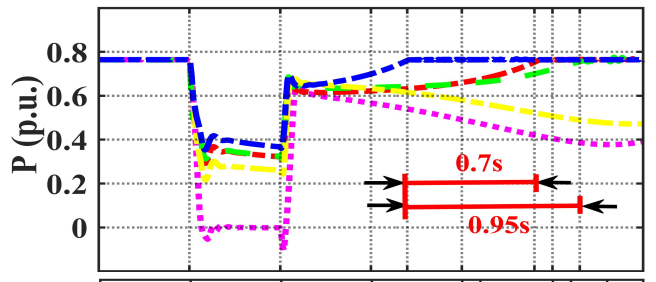

(b)

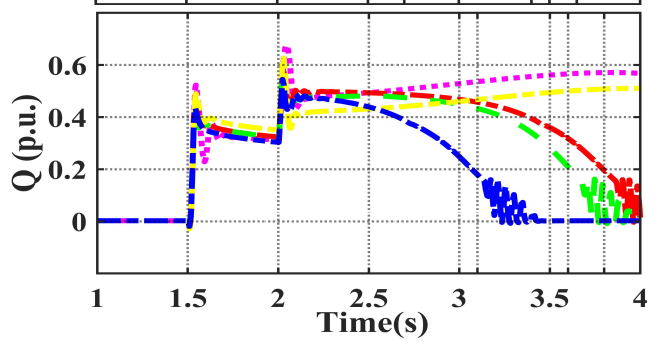

(c)

Fig. 7. (a) Dynamic voltage profile at bus 4 of MG_1 and injected (b) active, and (c) reactive power from the PV system for Fault__II.

is $0.7 \mathrm{~s}$ faster compared to Case_III and $0.5 \mathrm{~s}$ in comparison to Case_IV. This similar order can also be observed for the reactive power restoration to the normal operating condition from Fig. 7c. The reactive power decreases with the increment of voltage and restores to pre-fault value as the voltage is restored. However, the pattern of active power recovery to the pre-fault value is substantially different compared to the voltage recovery pattern for these three cases (Case_III, Case_IV, and Case_V). From Fig. 7b, it can be observed that for active power, Case_III provides earlier recovery than Case_IV though Case_IV recovered the voltage faster than Case_III. However, Case_V achieved accelerated recovery in both voltage and active power compared to Case_III and Case_IV. Case_V recovered the active power $0.7 \mathrm{~s}$ and $0.95 s$ faster compared to Case_III and Case_IV, respectively. This investigation conveys that voltage sensitivity based current injection by proposed strategy results enhanced dynamic voltage stability along with accelerated active power recovery following fault occurrence.

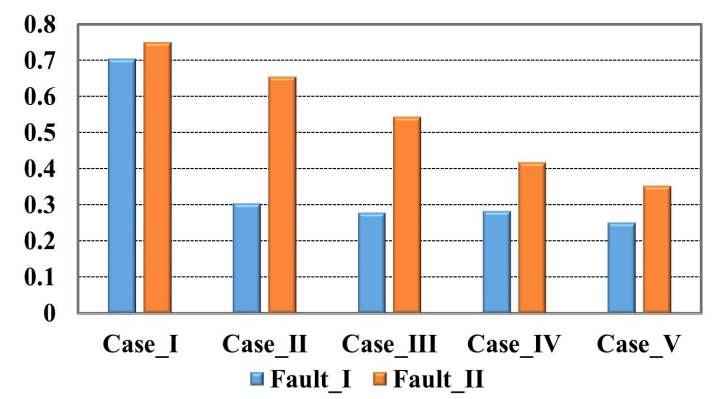

Fig. 8. VSSIs at load bus of MG_1 for Fault_I and Fault_II.

The measured VSSIs for both faults at load bus for all cases are presented in Fig. 8. As can be seen in the Fault_I scenario, VSSI is very high for Case_I compared to other cases. This is due to the voltage instability for Case_I. Although all other cases

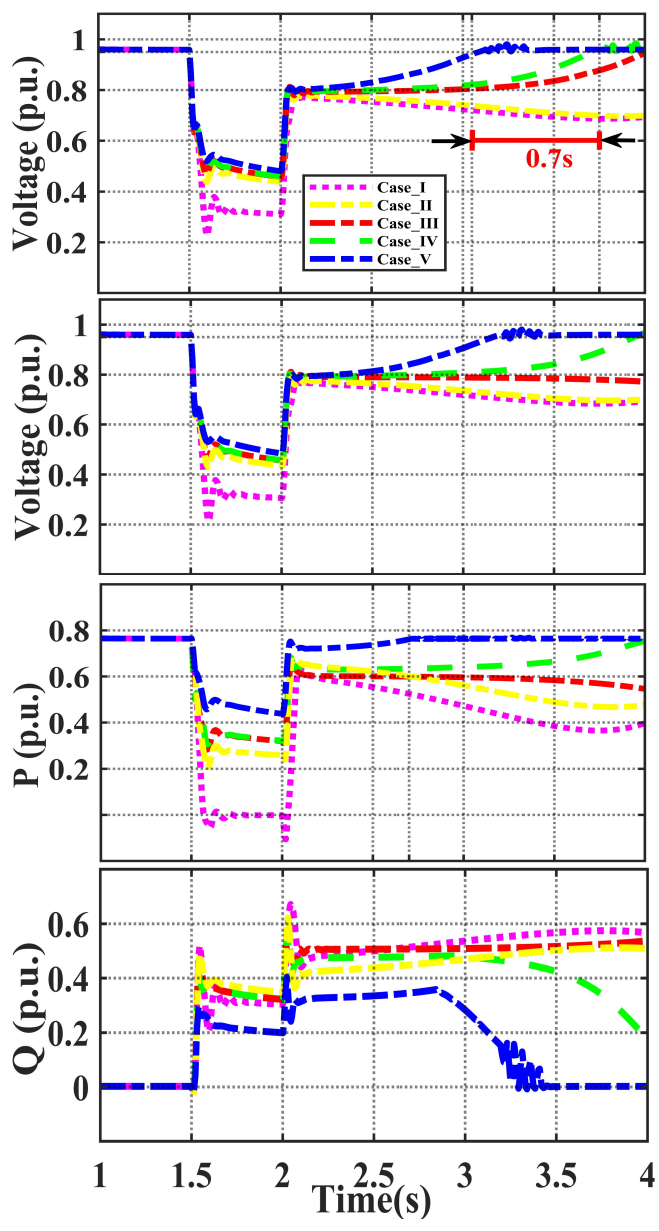

(a)

(b)

(c)

Fig. 9. Dynamic voltage profile at bus 4 of $M G_{-} 1$ for Fault_II with (a) R/X=1.74; (b) $\mathrm{R} / \mathrm{X}=2.22$; and injected (c) active and (d) reactive power with $\mathrm{R} / \mathrm{X}=2.22$.

(Case_II to Case_V) recovered the voltage, there are moderately different VSSIs because various cases have different VRTs. On the other hand, for Fault_II, Case_II also follows a similar pattern to Case_I after fault clearance. However, Case_II has a lower voltage drop during fault. As a result, the VSSI for Case_II is lower than Case_I but higher than others owing to the voltage instability. Since only Case_III, Case_IV, and Case_V was able to recover the voltage, they have lower VSSI. However, Case_V represents the lowest VSSI among all strategies. As lower VSSI demonstrates improved DVS, highest performance in terms of DVS augmentation is achieved with the proposed strategy. This is also in alignment with the dynamic simulations.

2) Different $R / X$ ratio: In this section, the DVS is investigated with the increment of the $R / X$ ratio of the network. The dynamic voltage profiles at bus 4 with the $R / X$ ratio of 1.74 and 2.22 for Fault_II are depicted in Fig. 9a and 9b, respectively. Both Case_I and Case_II have experienced instability in these scenarios which are similar to the previous investigation. For Case_III, the dynamic voltage profile is deteriorated with the increment of the $\mathrm{R} / \mathrm{X}$ ratio. Case_III with $R / X=1.74$ suffers from increased VRT than the previous investigation of Fig. 7 a where $R / X$ ratio is 1.31 , then voltage instability occurs for $R / X=2.22$. In a similar pattern, Case_IV encounters increased VRT with the increment of $R / X$ ratio. In contrast, VRT is almost invariant for Case_V even though the $R / X$ ratio is increased. As a consequence, the differences between the VRTs of Case_V and other cases have increased with the increment of $R / X$ ratio. This is because Case_V provides the 


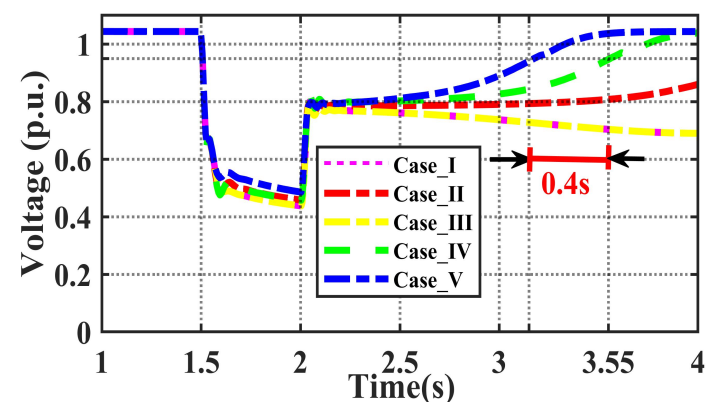

Fig. 10. Dynamic voltage profile at bus 4 with power factor 0.98 operation of PV system for Fault_II.

consistent voltage recovery with the increment of $\mathrm{R} / \mathrm{X}$ ratio while other strategies observed slower recovery. Active and reactive power recovery for $\mathrm{R} / \mathrm{X}$ ratio of 2.2 are shown in Fig. 9c and 9d, respectively. As observed, only Case IV and V was able to recover the active power within the considered time frame. However, Case $\mathrm{V}$, the proposed strategy, have realized the fastest recovery than Case IV (around 1.3sec faster). Finally enhanced DVS by the proposed strategy occurs because of the determination of the current components based on voltage sensitivity coefficients, which makes it distinct from that of other strategies.

3) Different Power Factor Operation: To investigate the effectiveness of the proposed strategy further, 0.98 power factor (pf) operating condition of the PV system is considered in this section. The dynamic voltage at bus 4 for this scenario with Fault_II and $R / X=2.22$ condition is illustrated in Fig. 10. The voltage profile is improved compared to the previous studies at the normal condition due to 0.98 pf operation. As a consequence, the voltage depth during fault is reduced for all cases. DVS performance in Case_III, Case_IV, and Case_V is improved at 0.98 pf compared with their respective performances with unity pf (presented in Section IV-A2). The VRT for Case_IV and Case_V is $3.55 \mathrm{~s}$ and $3.15 \mathrm{~s}$, respectively in this scenario. Nevertheless, Case_I to Case_III was unable to recover the voltage. Finally, the most obvious finding to emerge from the analysis is that Case $\mathrm{V}$ still provides considerably improved DVS compared with other strategies, and specifically, $0.4 s$ faster VRT than Case_IV.

4) Unbalanced Fault: In this section, a two lines to ground (2LG) fault is investigated. The three-phase voltage dynamics at bus 4 for the 2LG fault are illustrated in Fig.11. Witnessed by Phase A and B voltages from Fig.11a and 11b, respectively, the voltages have been retrieved to the stable condition $(0.95 \mathrm{pu})$ in Case_III, Case_IV, and Case_V. For Case_I and Case_II, the voltage recovery is in an upward direction but has not recovered within the considered time frame. The voltage recovery-speed is enhanced on both Phase A and Phase B with the proposed strategy in Case_V compared to other cases. As shown in Fig.11c, rising voltage swell on Phase $\mathrm{C}$ can also be observed in an identical order. However, the voltage swell is very insignificant which is also within the over voltage limit (1.1pu). Finally, it can be concluded that the proposed method of injecting active and reactive current is also effective for enhancing the DVS following an unsymmetrical disturbance.

\section{B. Microgrid System_2}

The second MG system (MG_2) is the modified IEEE 15 bus system consisting of 1.4 MVA total load with the equal combination of ZIP (50\%) and IM load (50\%) [25]. Detailed loading data is given in Table IV of Appendix. Two PV systems with a capacity of 500kVA each, a battery energy storage of 1.4 MVA, and a backup diesel generator of 1.5 MVA are installed as shown in Fig. 12. Fault_I and Fault_II are applied at the point F of MG_2. The cases as presented in Table I are also investigated for MG_2. Since bus 5 is the farthest bus, dynamic voltage at bus 5 is examined. Fig. 13a and 13b illustrate the dynamic voltage at bus 5 for Fault_I and Fault_II, respectively. The voltage instability occurs with Case_I and Case_II for both faults, while instability occurs with only Fault_II scenario for Cases_III and Case_IV. In contrast, Case_V is capable of resisting the instability for both faults. For Fault_I condition, though Case_III, Case_IV, and Case_V recovered the voltage, the difference in VRT between Case_V and the other two cases, Case_III and Case_IV, are $0.73 s$ and $0.43 s$, respectively. The enhanced performance of the proposed strategy also persists in the Fault_II condition. The prominent performance by Case_V on augmenting DVS is shown in Fig. 13b where only Case_V was able to secure the DVS of MG_2 following the fault among all the strategies. The obtained VSSIs at different buses of MG_2 for different cases for Fault_2 are shown in Fig. 14. Since MG_2 experiences an enhanced DVS for Case_V only, the difference between the VSSI of Case_V and other cases are significant. These results confirm that an enhanced DVS operation of MGs can be achieved by implementing the proposed VS strategy.

\section{CONCLuSion}

In this paper, a VS strategy based on voltage sensitivity subject to active and reactive current is proposed. Voltage sensitivity assessment conveys the correlation of voltage with the injection of active and reactive current components. The variation of voltage sensitivity coefficients with the variation of the $R / X$ ratio is analyzed. It is observed that the voltage sensitivity with respect to active current increases with the increment of the $R / X$ ratio. Therefore, proposed strategy injects both the active and reactive current based on the voltage sensitivity coefficients. To evaluate the effectiveness, the proposed strategy is compared with other strategies which also inject both active and reactive current, and validated in two islanded MGs. Different scenarios, such as symmetrical and asymmetrical faults, various $R / X$ ratio, different pf operation are investigated. The results show that the proposed strategy provides faster voltage recovery for all the scenarios compared to considered existing strategies and can resist probable instability. In addition, accelerated active power recovery is also achieved by the proposed strategy. The VSSIs also demonstrate that the proposed strategy experiences the lowest voltage recovery time. In conclusion, proposed strategy is capable of providing enhanced DVS and faster active power reattainment following fault of islanded MGs.

\section{REFERENCES}

[1] M. Eskandari, L. Li, M. H. Moradi, F. Wang, and F. Blaabjerg, "A control system for stable operation of autonomous networked microgrids," IEEE Transactions on Power Delivery, 2019.

[2] M. Farrokhabadi, C. A. Cañizares, J. W. Simpson-Porco, E. Nasr, L. Fan, P. A. Mendoza-Araya, R. Tonkoski, U. Tamrakar, N. Hatziargyriou, D. Lagos et al., "Microgrid stability definitions, analysis, and examples," IEEE Transactions on Power Systems, vol. 35, no. 1, pp. 13-29, 2019.

[3] N. Afrin, F. Yang, and J. Lu, "Dynamic voltage stability improvement of islanded microgrid by smart pv inverter," in 2019 IEEE PES Asia-Pacific Power and Energy Engineering Conference (APPEEC). IEEE, 2019, pp. $1-5$. 


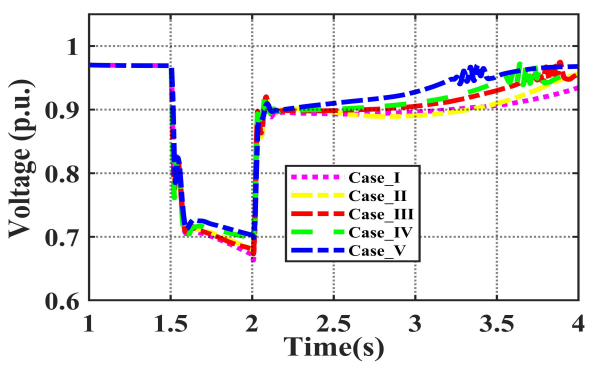

(a)

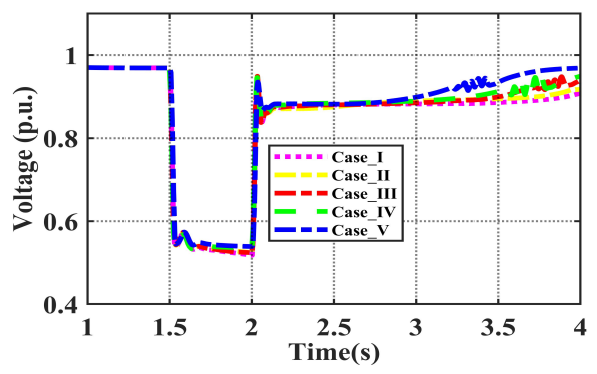

(b)

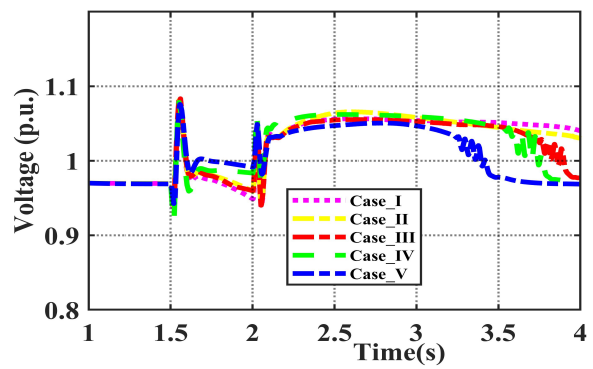

(c)

Fig. 11. Dynamic voltage profile at (a) Phase A, (b) Phase B, and (c) Phase C for 2LG fault at bus 4 of MG_1.

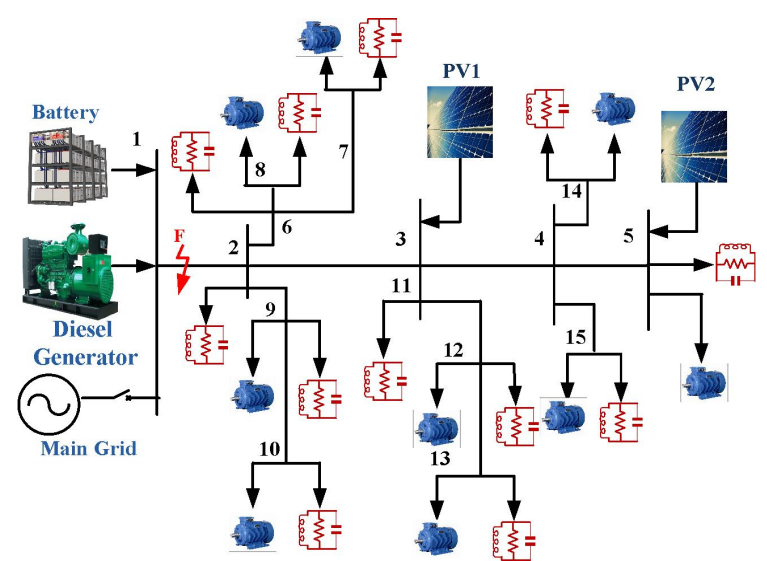

Fig. 12. Modified IEEE 15-bus system as Microgrid system_2.

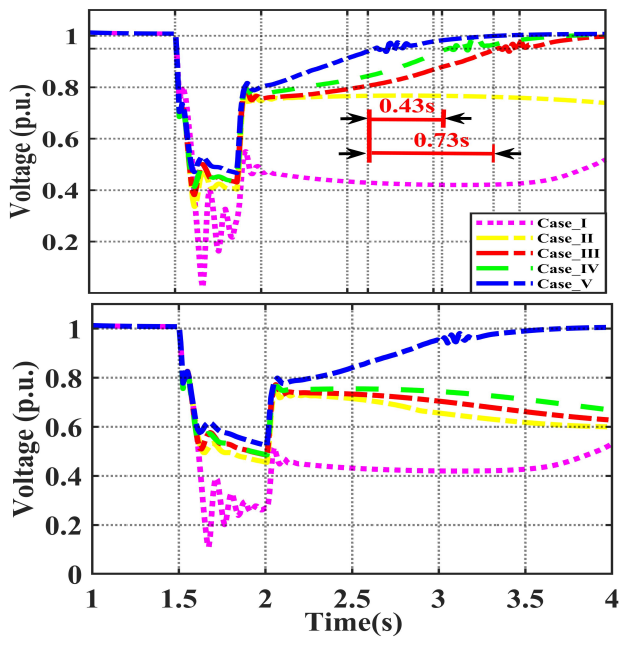

Fig. 13. Dynamic voltage profile at bus 5 of MG_2 for (a) Fault_I and (b) Fault_II.

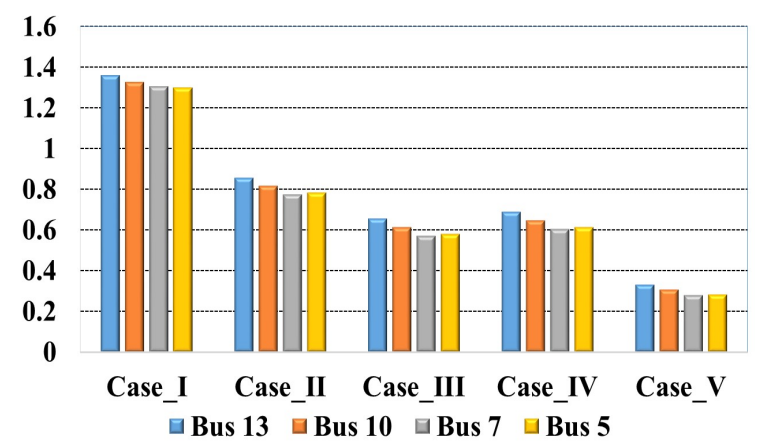

Fig. 14. VSSIs at different buses of MG_2 for different cases for Fault_II.
[4] L. A. de Souza Ribeiro, O. R. Saavedra, S. L. De Lima, and J. G. De Matos, "Isolated micro-grids with renewable hybrid generation: The case of lençóis island," IEEE Transactions on sustainable energy, vol. 2, no. 1, pp. 1-11, 2010.

[5] W.-M. Guo, L.-H. Mu, and X. Zhang, "Fault models of inverter-interfaced distributed generators within a low-voltage microgrid," IEEE Transactions on Power Delivery, vol. 32, no. 1, pp. 453-461, 2016.

[6] N. Afrin, F. Yang, and J. Lu, "Voltage support strategy for pv inverter to enhance dynamic voltage stability of islanded microgrid," International Journal of Electrical Power \& Energy Systems, vol. 121, p. 106059, 2020.

[7] A. K. Alaboudy, H. H. Zeineldin, and J. Kirtley, "Microgrid stability characterization subsequent to fault-triggered islanding incidents," IEEE transactions on power delivery, vol. 27, no. 2, pp. 658-669, 2012.

[8] M. Islam, N. Mithulananthan, and M. Hossain, "Dynamic voltage support by tl-pv systems to mitigate short-term voltage instability in residential dn," IEEE Transactions on Power Systems, vol. 33, no. 4, pp. 4360-4370, 2018.

[9] M. A. Hossain, H. R. Pota, M. J. Hossain, and F. Blaabjerg, "Evolution of microgrids with converter-interfaced generations: Challenges and opportunities," International Journal of Electrical Power \& Energy Systems, vol. 109, pp. 160-186, 2019.

[10] N. Afrin, F. Yang, J. Lu, and M. Islam, "Impact of induction motor load on the dynamic voltage stability of microgrid," in 2018 Australian \& New Zealand Control Conference (ANZCC). IEEE, 2018, pp. 397-402.

[11] W. Wang and F. De Leon, "Quantitative evaluation of der smart inverters for the mitigation of fidvr in distribution systems," IEEE Transactions on Power Delivery, vol. 35, no. 1, pp. 420-429, 2019.

[12] A. Q. Al-Shetwi, M. Z. Sujod, and N. L. Ramli, "A review of the fault ride through requirements in different grid codes concerning penetration of pv system to the electric power network," ARPN journal of engineering and applied sciences, vol. 10, no. 21, pp. 9906-9912, 2015.

[13] E. Storage, "Ieee standard for interconnection and interoperability of distributed energy resources with associated electric power systems interfaces amendment 1: To provide more."

[14] I. I. Perpinias, N. P. Papanikolaou, and E. C. Tatakis, "Optimum design of low-voltage distributed photovoltaic systems oriented to enhanced fault ride through capability," IET Generation, Transmission \& Distribution, vol. 9, no. 10, pp. 903-910, 2015.

[15] M. Brenna, E. De Berardinis, L. D. Carpini, F. Foiadelli, P. Paulon, P. Petroni, G. Sapienza, G. Scrosati, and D. Zaninelli, "Automatic distributed voltage control algorithm in smart grids applications," IEEE Transactions on Smart Grid, vol. 4, no. 2, pp. 877-885, 2012.

[16] Y. Yang, H. Wang, and F. Blaabjerg, "Reactive power injection strategies for single-phase photovoltaic systems considering grid requirements," IEEE Transactions on Industry Applications, vol. 50, no. 6, pp. 4065-4076, 2014.

[17] M. Islam, N. Mithulananthan, and M. Hossain, "Dynamic voltage support by tl-pv systems to mitigate short-term voltage instability in residential dn," IEEE Transactions on Power Systems, vol. 33, no. 4, pp. 4360-4370, 2017.

[18] N. Afrin, F. Yang, and J. Lu, "Optimized reactive power support strategy for pv inverter to intensify the dynamic voltage stability of islanded microgrid," International Transactions on Electrical Energy Systems, vol. v, p. PP, 2020, doi:10.1002/2050-7038.12356.

[19] M. Islam, M. Nadarajah, and J. Hossain, "Short-term voltage stability enhancement in residential grid with high penetration of rooftop pv units," IEEE Transactions on Sustainable Energy, 2018.

[20] K. Kawabe, Y. Ota, A. Yokoyama, and K. Tanaka, "Novel dynamic voltage support capability of photovoltaic systems for improvement of short-term voltage stability in power systems," IEEE Transactions on Power Systems, vol. 32, no. 3, pp. 1796-1804, 2016.

[21] M. S. Ayaz, R. Azizipanah-Abarghooee, and V. Terzija, "European lv microgrid benchmark network: Development and frequency response analysis," in 2018 IEEE International Energy Conference (ENERGYCON). IEEE, 2018, pp. 1-6. 
[22] R. Aghatehrani and R. Kavasseri, "Sensitivity-analysis-based sliding mode control for voltage regulation in microgrids," IEEE Transactions on Sustainable Energy, vol. 4, no. 1, pp. 50-57, 2012.

[23] S. Qazi, "Chapter 2 - fundamentals of standalone photovoltaic systems," in Standalone Photovoltaic (PV) Systems for Disaster Relief and Remote Areas, S. Qazi, Ed. Elsevier, 2017, pp. 31 - 82. [Online]. Available: http://www.sciencedirect.com/science/article/pii/B9780128030226000022

[24] Y. Dong, X. Xie, B. Zhou, W. Shi, and Q. Jiang, "An integrated high side varvoltage control strategy to improve short-term voltage stability of receivingend power systems," IEEE Transactions on Power Systems, vol. 31, no. 3, pp. 2105-2115, 2015.

[25] Y. Zheng, Z. Y. Dong, Y. Xu, K. Meng, J. H. Zhao, and J. Qiu, "Electric vehicle battery charging/swap stations in distribution systems: comparison study and optimal planning," IEEE transactions on Power Systems, vol. 29, no. 1, pp. 221-229, 2013.

[26] C. W. Taylor, "Power system voltage stability," McGraw-Hill, 1994.

\section{APPENDIX}

TABLE II

PU PARAMETERS OF IM LOAD [26]

\begin{tabular}{|l|l|l|l|l|l|l|}
\hline$R_{S}$ & $X_{s}$ & $R_{r}$ & $X_{r}$ & $X_{m}$ & $H$ & $\mathrm{p}$ \\
\hline 0.1 & 0.1 & 0.09 & 0.06 & 2.5 & 0.28 & 2 \\
\hline
\end{tabular}

TABLE III

LOAD OF MICROGRID SYSTEM_1

\begin{tabular}{|c|cc|cc|}
\hline \multirow{2}{*}{ Phase } & \multicolumn{2}{|c|}{ ZIP } & \multicolumn{2}{c|}{ IM } \\
\cline { 2 - 5 } & $\mathrm{kW}$ & $\mathrm{kVAr}$ & $\mathrm{kW}$ & $\mathrm{kVAr}$ \\
\hline $\mathrm{A}$ & 150 & 72.70 & 150 & 72.63 \\
\hline $\mathrm{B}$ & 150 & 72.70 & 150 & 72.63 \\
\hline $\mathrm{C}$ & 150 & 72.70 & 150 & 72.63 \\
\hline
\end{tabular}

TABLE IV

LOAD OF MICROGRID SYSTEM_2

\begin{tabular}{|l|lll|lll|llll|lll|l|}
\hline \multirow{2}{*}{ Bus } & \multicolumn{9}{|c|}{ ZIP } & \multicolumn{3}{|c|}{ KW } & \multicolumn{3}{c|}{ kVAr } & \multicolumn{5}{c|}{ KW } & \multicolumn{3}{c|}{ kVAr } \\
\hline & A & B & C & A & B & C & A & B & C & A & B & C \\
\hline 2 & 8.75 & 8.75 & 8.75 & 11.25 & 11.25 & 11.25 & 5.95 & 5.95 & 5.95 & 3.75 & 3.75 & 3.75 \\
\hline 3 & 6.33 & 6.33 & 6.33 & 4.36 & 4.36 & 4.36 & 17 & 17 & 17 & 10.7 & 10.7 & 10.7 \\
\hline 4 & 6.91 & 6.91 & 6.91 & 8.64 & 8.64 & 8.64 & & & & & & \\
\hline 5 & 8.75 & 8.75 & 8.75 & 11.25 & 11.25 & 11.25 & 5.95 & 5.95 & 5.95 & 3.75 & 3.75 & 3.75 \\
\hline 6 & 6.91 & 6.91 & 6.91 & 8.64 & 8.64 & 8.64 & 29.65 & 29.65 & 29.65 & 18.66 & 18.66 & 18.66 \\
\hline 7 & 14.28 & 14.28 & 14.28 & 19.23 & 19.23 & 19.23 & 17 & 17 & 17 & 10.7 & 10.7 & 10.7 \\
\hline 8 & 25.41 & 25.41 & 25.41 & 34.23 & 34.23 & 34.23 & 21.25 & 21.25 & 21.25 & 13.37 & 13.37 & 13.37 \\
\hline 9 & 6.33 & 6.33 & 6.33 & 13.10 & 13.10 & 13.10 & 17 & 17 & 17 & 10.7 & 10.7 & 10.7 \\
\hline 10 & 8.75 & 8.75 & 8.75 & 11.25 & 11.25 & 11.25 & 5.95 & 5.95 & 5.95 & 3.75 & 3.75 & 3.75 \\
\hline 11 & 6.33 & 6.33 & 6.33 & 13.10 & 13.10 & 13.10 & 17 & 17 & 17 & 10.7 & 10.7 & 10.7 \\
\hline 12 & 8.75 & 8.75 & 8.75 & 11.25 & 11.25 & 11.25 & 5.95 & 5.95 & 5.95 & 3.75 & 3.75 & 3.75 \\
\hline 13 & 12.75 & 12.75 & 12.75 & 17.12 & 17.12 & 17.12 & 21.25 & 21.25 & 21.25 & 13.37 & 13.37 & 13.37 \\
\hline 14 & 12.75 & 12.75 & 12.75 & 17.12 & 17.12 & 17.12 & 21.25 & 21.25 & 21.25 & 13.37 & 13.37 & 13.37 \\
\hline 15 & 6.33 & 6.33 & 6.33 & 13.10 & 13.10 & 13.10 & 17 & 17 & 17 & 10.70 & 10.70 & 10.70 \\
\hline
\end{tabular}

